\title{
Dual specificity phosphatase 1 has a protective role in osteoarthritis fibroblast-like synoviocytes via inhibition of the MAPK signaling pathway
}

\author{
HUA-ZHI PENG ${ }^{*}$, ZHE YUN* ${ }^{*}$ WEI WANG and BAO-AN MA \\ Department of Orthopaedics, Tangdu Hospital, The Fourth Military Medical University, Xi'an, Shanxi 710038, P.R. China
}

Received December 12, 2016; Accepted August 10, 2017

DOI: $10.3892 / \mathrm{mmr} .2017 .7617$

\begin{abstract}
Increasing evidence indicates the important role of inflammation in the pathogenesis and progression of osteoarthritis (OA). Dual specificity phosphatase 1 (DUSP1), a negative regulator of the mitogen-activated protein kinase (MAPK) signaling pathway, has anti-inflammatory properties. In the present study, the expression of DUSP1 was investigated in human OA fibroblast-like synoviocytes (FLSs), human normal FLSs and OA FLSs pretreated with dexamethasone at the mRNA and protein levels. Then, the activation of MAPK pathway proteins and the expression of matrix metalloproteinase-13 (MMP-13) and cyclooxygenase-2 (COX-2) were measured by western blot analysis in the three groups of cells. Dexamethasone induced the expression of DUSP1 and inhibited the activation of the MAPK pathway and reduced the expression of MMP-13 and COX-2 in OA FLSs. However, the role of DUSP1 remained unclear. To clarify this, the effects of overexpression of DUSP1 in OA FLSs were determined using a DUSP1-overexpressing lentivirus. The results demonstrated that overexpression of DUSP1 in OA FLSs inhibited the activation of the MAPK pathway and expression of OA-associated mediators. The findings of the present study indicate that DUSP1 has a protective role in OA FLSs and may be a potential target in the treatment of OA.
\end{abstract}

\section{Introduction}

Osteoarthritis (OA) is the most common form of joint disease in the world and constitutes a major cause of disability in the aging population (1). The disease was previously considered

Correspondence to: Professor Bao-An Ma, Department of Orthopaedics, Tangdu Hospital, The Fourth Military Medical University, 569 Xinsi Road, Baqiao, Xi'an, Shanxi 710038, P.R. China

E-mail: mabaoan2016@126.com

${ }^{*}$ Contributed equally

Key words: dual specificity phosphatase1, mitogen activated protein kinase, osteoarthritis, fibroblast-like synoviocytes to be a typical non-inflammatory arthropathy, but currently it is generally accepted that it is an inflammatory disease (2). Previous studies have reported that inflammation contributes to the symptoms and the progression of OA $(3,4)$. Furthermore, certain researchers have observed that synovial inflammation is present in the earlier phases of OA prior to visible cartilage degeneration (5-7). These studies suggest that disease-modifying interventions targeting inflammatory processes may be effective for the prevention and treatment of OA.

The mitogen-activated protein kinase (MAPK) signaling pathway is present in all eukaryotes and has a major role in various inflammatory diseases. The members of this signaling pathway group include p38 MAPK, c-Jun NH2 terminal kinase (JNK) and extracellular signal-regulated kinase. They are activated via phosphorylation of specific tyrosine and threonine residues by upstream factors. MAPKs regulate various physiological processes, including cell proliferation, differentiation, apoptosis and stress responses, and p38 and JNK are associated with the regulation of inflammatory and immune responses (8-11). The activation of MAPK is involved in the expression of several inflammatory genes, such as tumor necrosis factor (TNF), interleukin (IL)-1, IL-6, cyclooxygenase-2 (COX-2) and certain enzymes such as inducible nitric oxide synthase and matrix metalloproteinase (MMP)-13 (12-15).

Dual specificity phosphatase 1 (DUSP1; also termed MAPK phosphatase 1) is one of an 11-member family that inhibits the activity of MAPKs by dephosphorylating tyrosine and threonine residues at the MAPK Thr-Xaa-Tyr activation motif. It is a particularly effective inhibitor of JNK and p38 MAPK signaling pathways (16). DUSP1 is a nuclear phosphatase widely expressed in various tissues, and its expression is regulated by a wide variety of different stimuli, including cellular stress, cytokines, lipopolysaccharide and glucocorticoids (17-20). Several studies have demonstrated that DUSP1 is an important negative regulator of inflammatory responses (21-26), and the induction of DUSP1 gene expression is potentially a novel anti-inflammatory strategy. However, to date, the role of DUSP1 in human OA synovial inflammation and its molecular mechanisms remain unclear.

The current study investigated the expression of DUSP1 in cultured human normal fibroblast-like synoviocytes (FLSs) and OA FLSs, and the effect of DUSP1 on the expression of OA-associated mediators, such as MMP-13 and COX-2, which 
occurs through a mechanism involving the inhibition of the p38 MAPK/JNK signaling pathway. Dexamethasone was used induce the expression of DUSP1 in OA FLSs, which partially demonstrated the anti-inflammatory mechanism of glucocorticoids in OA. The results demonstrated the anti-inflammatory and anti-catabolic actions of DUSP1 on OA FLSs, and DUSP1 was a potential target of treatment in OA.

\section{Materials and methods}

Reagents. Reagents were purchased from Sigma-Aldrich (Merck KGaA, Darmstadt, Germany) unless otherwise stated. Dexamethasone was purchased from Shanghai GeneChem Co, Ltd. (cat. no. A601187-0005; Shanghai, China). Antibodies against DUSP1 (cat. no. AF1343a), COX-2 (cat. no. AJ1195b), MMP-13 (cat. no. AP13706c), phosphorylated (p-) JNK (cat. no. AB208035), JNK (cat. no. AB4821) and p38 MAPK (cat. no. AJ1201a) were purchased from Abgent, Inc. (San Diego, CA, USA). Antibody against p-p38 MAPK was obtained from Santa Cruz Biotechnology, Inc. (cat. no. sc101759; Dallas, TX, USA). Antibodies against $\beta$-tubulin (cat. no. ab6406) and GAPDH (cat. no. ab8245) were purchased from Abcam (Cambridge, MA, USA).

Specimen selection and cell culture. Human OA and normal synovial tissue specimens were obtained from patients with OA requiring joint replacement surgery and trauma patients undergoing post-traumatic amputation from June 2015 to June 2016, respectively. In the OA patients, two patients were male and three patients were female, the age range was 57-76 years and the mean age \pm standard error of the mean (SEM) was $65.2 \pm 3.2$ years. All three trauma patients were male, the age range was $22-44$ years and the mean age $( \pm$ SEM) was $31.0 \pm 5.9$ years. Informed consent was obtained from patients for the use of their tissues for research purposes. The present study was approved by the Ethics Committee of Tangdu Hospital, Fourth Military Medical University, (Xi'an, China). Tissues were carefully minced and digested with $0.2 \%$ collagenase I in Dulbecco's modified Eagle's medium (DMEM; (Hyclone; GE Healthcare Life Sciences, Logan, UT, USA) for 4-6 h at $37^{\circ} \mathrm{C}$, filtered through a 200 -mesh sieve, and finally cultured in DMEM supplemented with $10 \%$ fetal bovine serum (Hyclone; GE Healthcare Life Sciences), 100 units penicillin and $100 \mu \mathrm{g} / \mathrm{ml}$ streptomycin. The cells were cultured up to $90 \%$ confluence and then split in a 1/3 ratio up to passage 3-6. FLSs at these passages were identified by flow cytometry as described previously (27) and used for subsequent experiments.

Reverse transcription-quantitative polymerase chain reaction $(R T-q P C R)$. Total RNA was isolated with TRIzol reagent (Invitrogen; Thermo Fisher Scientific, Inc, Waltham, MA, USA) according to the manufacturer's instructions. The cDNA was synthesized using a Reverse Transcription kit (Thermo Fisher Scientific, Inc.) and then was diluted 1:4 with RNase-free water. cDNA $(1 \mu \mathrm{l})$ was subjected to PCR analysis using SYBR Green Master Mix (Invitrogen; Thermo Fisher Scientific, Inc.) with a Motor-Gene Q RT-PCR instrument (Qiagen GmbH, Hilden, Germany). The primers were purchased from Sangon Biotech Co, Ltd. (Shanghai, China) and the sequences were as follows: Human DUSP1, 5'-AGTACCCCACTCTACGAT
CAGG-3' (forward) and 5'-GAAGCGTGATACGCACTGC-3' (reverse); human MMP-13, 5'-ACTGAGAGGCTCCGAGAA ATC-3' (forward) and 5'-GAACCCCGCATCTTGGCTT-3' (reverse); human COX-2, 5'-TTCAAATGAGATTGTGGG AAAATTGCT-3' (forward) and 5'-AGTTCATCTCTGCCT GAGTATCTT-3' (reverse); and $\beta$-actin, 5'-TAGTTGCGTTAC ACCCTTTCTTG-3' (forward) and 5'-TCACCTTCACCGTTC CAGTTT-3' (reverse). The conditions of PCR cycling were as follows: Denaturation step at $95^{\circ} \mathrm{C}$ for $10 \mathrm{~min}$, then 40 cycles at $95^{\circ} \mathrm{C}$ for $15 \mathrm{sec}, 65^{\circ} \mathrm{C}$ for $10 \mathrm{sec}$ and $72^{\circ} \mathrm{C}$ for $15 \mathrm{sec}$. Relative expression levels of target genes were calculated according to the $2^{-\Delta \Delta C q}$ method (28).

Western blot analysis. At the indicated time points, cultured FLSs were lysed in radioimmunoprecipitation containing proteinase inhibitor and phosphatase inhibitor and boiled. GAPDH and $\beta$-tubulin were used as internal reference protein. The protein was quantified by the bicinchoninic method. The extracted proteins $(20 \mu \mathrm{g} /$ well $)$ were loaded onto a $10 \%$ SDS-PAGE gel and electrophoresed for $2.5 \mathrm{~h}$ at $80 \mathrm{~V}$, then transferred to polyvinylidene fluoride (PVDF) membrane. PVDF membranes were blocked in 5\% skim milk in $1 \mathrm{X}$ Tris-buffered saline Tween (TBST) for $1 \mathrm{~h}$ at room temperature, washed with TBST, and the membranes were incubated for $24 \mathrm{~h}$ at $4^{\circ} \mathrm{C}$ with primary antibodies against DUSP1 (1:500), p38 (1:500), p-p38 (1:500), JNK (1:500), p-JNK (1:500), COX-2 (1:500), MMP-13 (1:500), GAPDH $(1: 3,000)$ or $\beta$-tubulin $(1: 3,000)$. Then, the PVDF membranes were washed in TBST and incubated with horseradish peroxidase (HRP)-labeled secondary antibodies for $1 \mathrm{~h}$ at $37^{\circ} \mathrm{C}$. The protein bands were detected using Immobilon Western Chemiluminescent HRP Substrate (EMD Millipore, Billerica, MA, USA) according to the manufacturer's instructions. Densitometry was performed using ImageJ software (Version 1.43; National Institutes of Health, Bethesda, USA).

Immunofluorescence staining. FLSs were washed with PBS, fixed with $4 \%$ paraformaldehyde, permeabilized with $0.2 \%$ Triton X-100 for $30 \mathrm{~min}$ at room temperature and then blocked with $5 \%$ bovine serum albumin for $1 \mathrm{~h}$ at room temperature. The cells were then incubated with DUSP1 polyclonal antibody (1:200) in 1\% bovine serum albumin (Thermo Fisher Scientific, Inc.) at $4^{\circ} \mathrm{C}$ overnight. The bound antibodies were detected by the cy3-conjugated secondary antibodies (1:3,000; cat. no. CW0159; CWBio, Inc, Beijing, China) for $2 \mathrm{~h}$ at room temperature. Cell nuclei were stained with DAPI $(50 \mu \mathrm{g} / \mathrm{ml})$ for $15 \mathrm{~min}$ at room temperature. The images were observed by inverted fluorescence microscope (Olympus Corporation, Tokyo, Japan).

Overexpression of DUSP1 in OA FLSs. The coding sequence of human DUSP1 was amplified by RT-PCR and ligated into the GV358 (Ubi-MCS-3FLAG-SV40-EGFP-IRES-puromycin) lentiviral vector (Shanghai GeneChem Co, Ltd, Shanghai, China) to produce LV-DUSP1. The GV358 was used as a negative control. The DUSP1 coding sequence was amplified from human genomic DNA by PCR using the primers: forwards, 5'-GAGGATCCCCGGGTACCGGTCGCCACCATGGTCAT GGAAGTGGGCAC-3' and reverse, 5'-TCCTTGTAGTCC ATACCGCAGCTGGGAGAGGTCGTAATG-3' . cDNA 
(10 ng/ $\mu \mathrm{l}, 1 \mu \mathrm{l}$ ) was subjected to PCR. The conditions of PCR cycling were as follows: Denaturation step at $98^{\circ} \mathrm{C}$ for $5 \mathrm{~min}$, then 30 cycles at $98^{\circ} \mathrm{C}$ for $10 \mathrm{sec}, 65^{\circ} \mathrm{C}$ for $10 \mathrm{sec}$ and $72^{\circ} \mathrm{C}$ for $60 \mathrm{sec}$. The GV358 vector was cleaved at the AgeI/AgeI site by a restriction endonuclease (10 units $/ \mu 1, \mathrm{NEB})$ at $37^{\circ} \mathrm{C}$ for $3 \mathrm{~h}$. The amplified DUSP1 gene was cloned into GV358 vector $(1 \mu \mathrm{g} / \mu \mathrm{l}, 2.5 \mu \mathrm{l})$ using PrimeSTAR HS DNA polymerase (cat. no. R010B; Takara Bio, Inc, Otsu, Japan) to generate LV-DUSP1. EndoFree midi Plasmid kit was purchased from Tiangen Biotech, Co, Ltd, (cat. no. DP118-2; Beijing, China). OA FLSs were infected with LV-DUSP1 and negative control lentivirus of equal titers $\left(1 \times 10^{8} \mathrm{TU} / \mathrm{ml}, \mathrm{MOI}=100\right)$ at $30 \%$ confluence and stable cells were selected with $2 \mu \mathrm{g} / \mathrm{ml}$ puromycin.

Statistical analysis. Data are presented as the mean \pm standard error. Statistically significant differences among three groups were identified by a one-way analysis of variance test followed by Tukey's post-hoc test. Differences between two groups were analyzed using Student's t test. All data were analyzed using SPSS version 19.0 (IBM Corp, Armonk, NY, USA). P<0.05 was considered to indicate a statistically significant difference.

\section{Results}

Expression of DUSP1 in OA FLSs, normal FLSs and dexamethasone (Dex)-induced OA FLSs. The mRNA expression of DUSP1 in OA FLSs, normal FLSs and OA FLSs pretreated with Dex $(1 \mu \mathrm{M})$ for $24 \mathrm{~h}$ were measured by RT-qPCR (Fig. 1A). The results demonstrated that the mRNA expression levels of DUSP1 in normal FLSs were significantly higher than in OA FLSs $(\mathrm{P}<0.01)$, and that Dex significantly induced DUSP1 expression in OA FLSs $(\mathrm{P}<0.01)$. The DUSP1 protein expression in the three groups was also detected by western blot and immunofluorescence staining (Fig. 1B and C). The findings verified the RT-qPCR results.

p38 MAPK and JNK activation, and the production of inflammatory and catabolic mediators in OA FLSs, normal FLSs and Dex-induced OA FLSs. It is well-established that the p38 MAPK and JNK are activated by phosphorylation. The level of p38, p-p38, JNK, p-JNK were determined by western blot in the three groups of cells (Fig. 2A and B). The results demonstrated that the expression of p-p38 and p-JNK in OA FLSs were higher than in normal FLSs and in OA FLSs pretreated with Dex $(\mathrm{P}<0.01)$, while the expression of $\mathrm{p} 38$ and JNK were decreased compared with normal FLSs and in OA FLSs pretreated with Dex, which indicated that Dex could inhibit the activation of p38 and JNK.

The expression of certain key mediators involved in OA, such as MMP-13 and COX-2, was also determined by western blot and RT-qPCR (Fig. 2C and D). The results suggested that the expression levels of MMP-13 and COX-2 in OA FLSs were higher than in normal FLSs and in OA FLSs pretreated with Dex $(\mathrm{P}<0.01)$, and partially demonstrated that the anti-inflammatory role of Dex in OA may act through a mechanism of MAPK signaling pathway inhibition.

Effect of DUSP1 overexpression in OA FLSs. To investigate the role of DUSP1 in OA FLSs, a lentivirus vector (GV358) combined with DUSP1 gene (LV-DUSP1) was used to infect

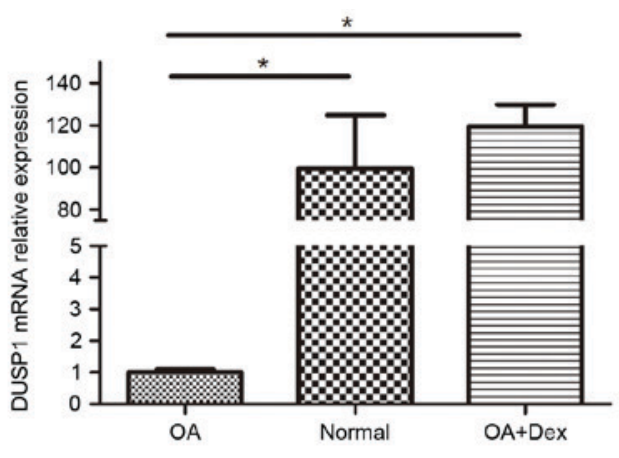

B
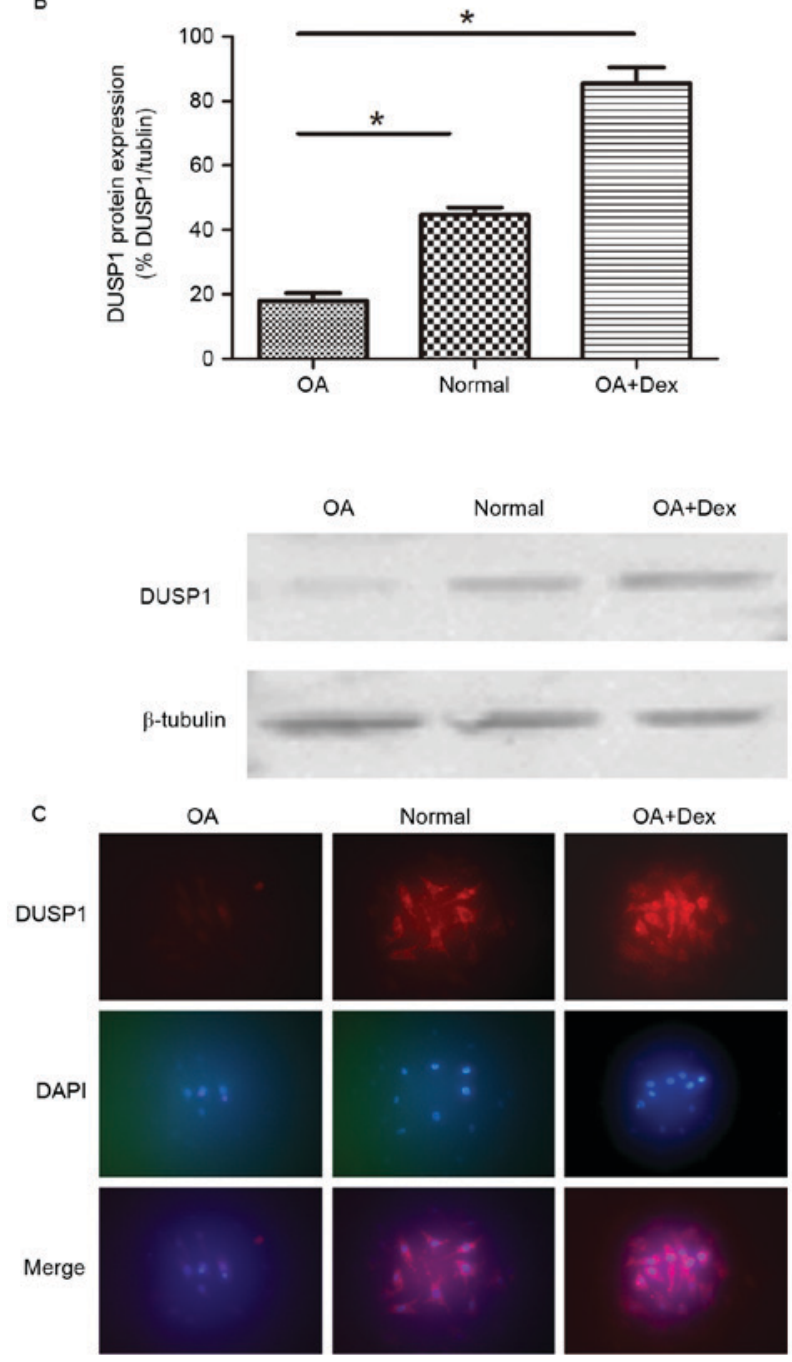

Figure 1. Expression of DUSP1 in OA FLSs, normal FLSs and Dex-induced OA FLSs. (A) DUSP1 mRNA expression levels were analyzed by reverse transcription-quantitative polymerase chain reaction (with $\beta$-actin as the reference gene). (B) DUSP1 protein expression levels were detected by western blot (with $\beta$-tubulin as the loading control). (C) Immunofluorescence staining detected DUSP1 protein expression (magnification, $\mathrm{x} 200$ ). Data are presented as the mean \pm standard error $(n=3)$. One-way analysis of variance test was performed for the comparison of the results. ${ }^{*} \mathrm{P}<0.01$. DUSP1, dual specificity phosphatase1; OA, osteoarthritis; FLSs, fibroblast-like synoviocytes; Dex, dexamethasone.

OA FLSs, and GV358 was used as a control. The infection efficiency was almost $90 \%$ (Fig. 3A). Western blot analysis confirmed DUSP1 overexpression (Fig. 3B). The activation 
A
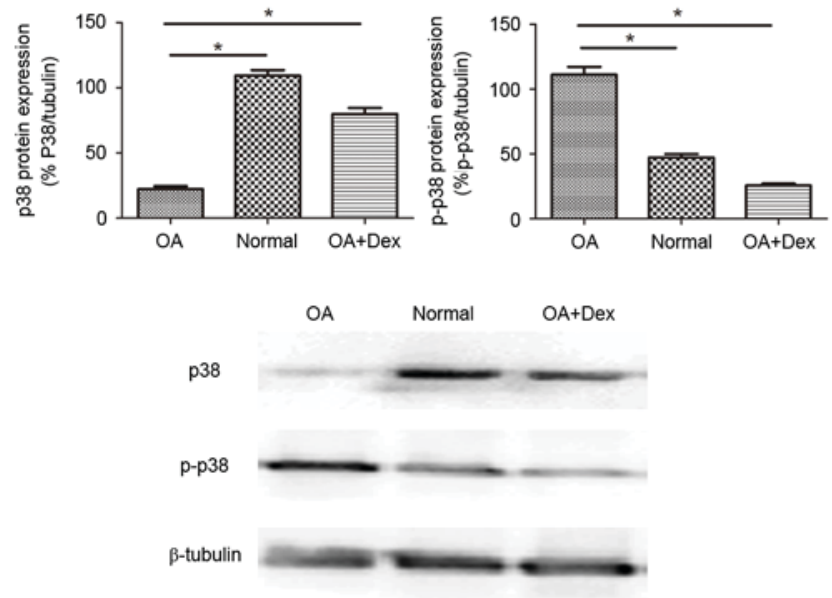

C
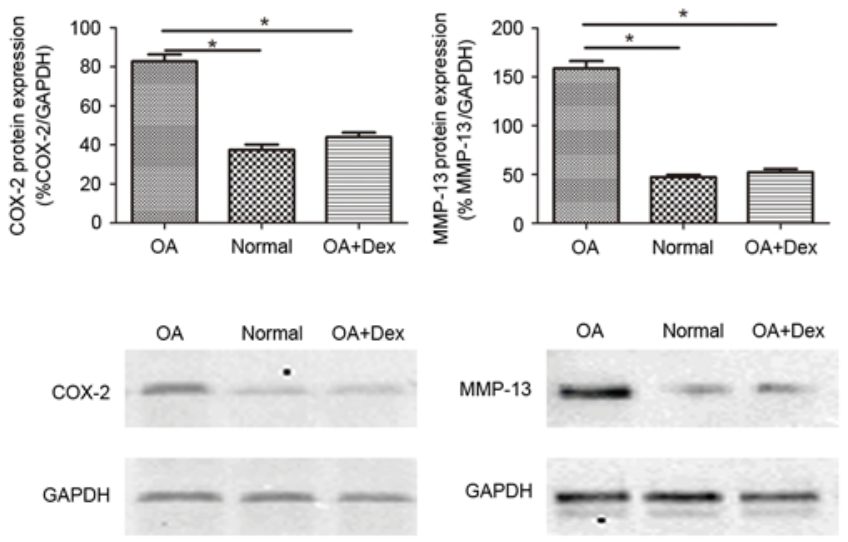
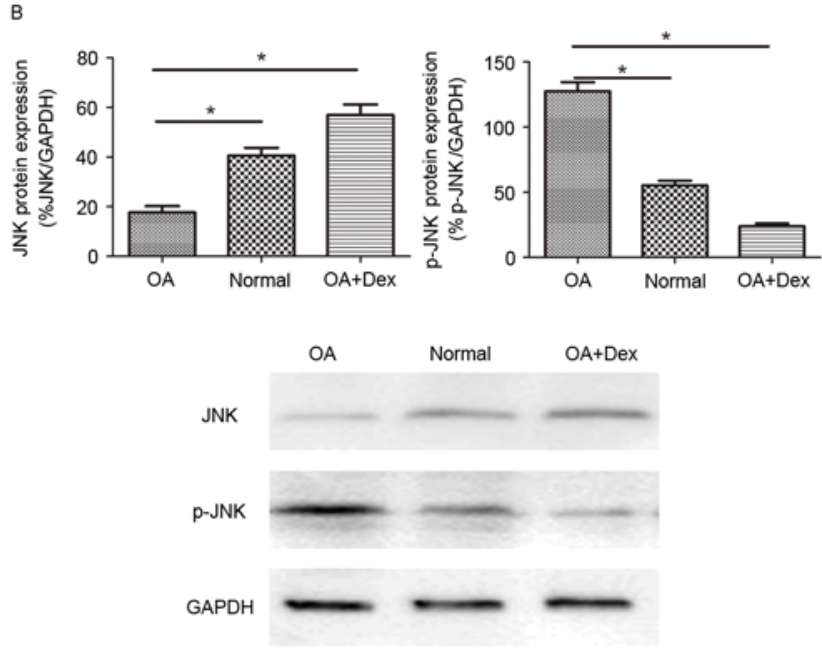

D
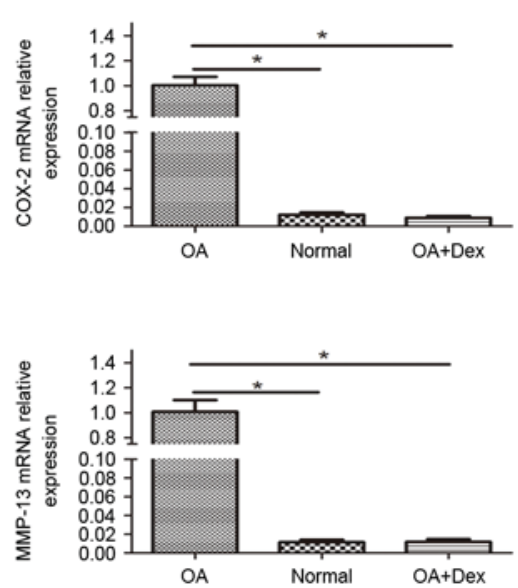

Figure 2. p38 MAPK and JNK pathway activation and the production of inflammatory and catabolic mediators in OA FLSs, normal FLSs and Dex-induced OA FLSs. The protein expression of (A) p38 MAPK, p-p38 MAPK, (B) JNK and p-JNK were measured by western blot with $\beta$-tubulin and GAPDH as loading controls, respectively. The expression of COX-2 and MMP-13 were detected by (C) western blot (with $\beta$-tubulin as the loading control) and (D) reverse transcription-quantitative polymerase chain reaction (with $\beta$-actin as the reference gene). One-way analysis of variance test was performed for the comparison of the results. Data are presented as the mean \pm standard error $(n=3)$. ${ }^{*} \mathrm{P}<0.01$. FLSs, fibroblast-like synoviocytes; MAPK, mitogen-activated protein kinase; OA, osteoarthritis; Dex, dexamethasone; p-, phosphorylated; JNK, c-Jun NH2 terminal kinase; COX-2, cyclooxygenase-2; MMP, matrix metalloproteinase.

of p38 MAPK and JNK pathways were detected by western blot. The results demonstrated that the expression of p-p38 and p-JNK were increased in the GV358 group compared with the LV-DUSP1 group. The expression of p38 and JNK was decreased in the GV358 group compared with the LV-DUSP1 group, which indicated that the activated p38 MAPK and JNK signaling pathway in OA FLSs was significantly inhibited by LV-DUSP1 (Fig. 3C). The expression levels of MMP-13 and COX-2 were also decreased in the presence of LV-DUSP1 (Fig. 3D). Taken together, these findings suggested that DUSP1 may inhibit inflammatory and catabolic mediators via inhibition of the p38 MAPK and JNK signaling pathway in OA FLSs.

\section{Discussion}

OA is one of the leading causes of physical disability (29). While there have been many surgical techniques, such as arthroplasty, used in the treatment of OA, there is currently no effective treatment to prevent or stop cartilage destruction. During the last several years, more and more research has demonstrated the important role of synovial inflammation in the pathogenesis of OA. Although there have been many inconclusive clinical results of exogenous anti-inflammatory therapy in OA (30). Anti-inflammatory treatment in OA, especially in the early stages, is a promising therapeutic approach to prevent cartilage degradation.

DUSP1 negatively regulates the MAPK signaling pathway by dephosphorylating MAPKs, and is involved in various cellular responses, including inflammation, cell proliferation, differentiation, stress responses, apoptosis and immune defense (19,31-33). The current study focused on the role of DUSP1 in OA, and the results demonstrated that DUSP1 has an anti-inflammation and anti-catabolic role, which is potentially mediated via inhibition of p38 MAPK and JNK signaling pathway in OA FLSs. These findings suggest that DUSP1 can be a potential target for the treatment of synovitis and preventing cartilage degradation in OA.

Initially, the DUSP1 mRNA and protein expression in OA FLSs and normal FLSs were determined, and increased expression levels of DUSP1in normal FLSs compared with in OA FLSs were observed. Glucocorticoids are powerful anti-inflammatory agents that reduce the expression of 


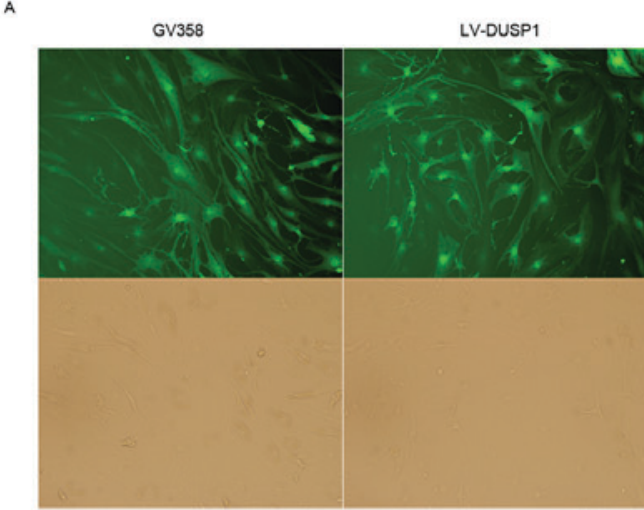

c
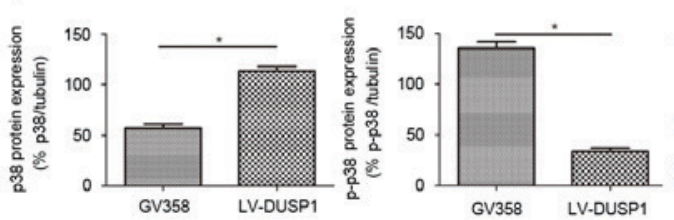
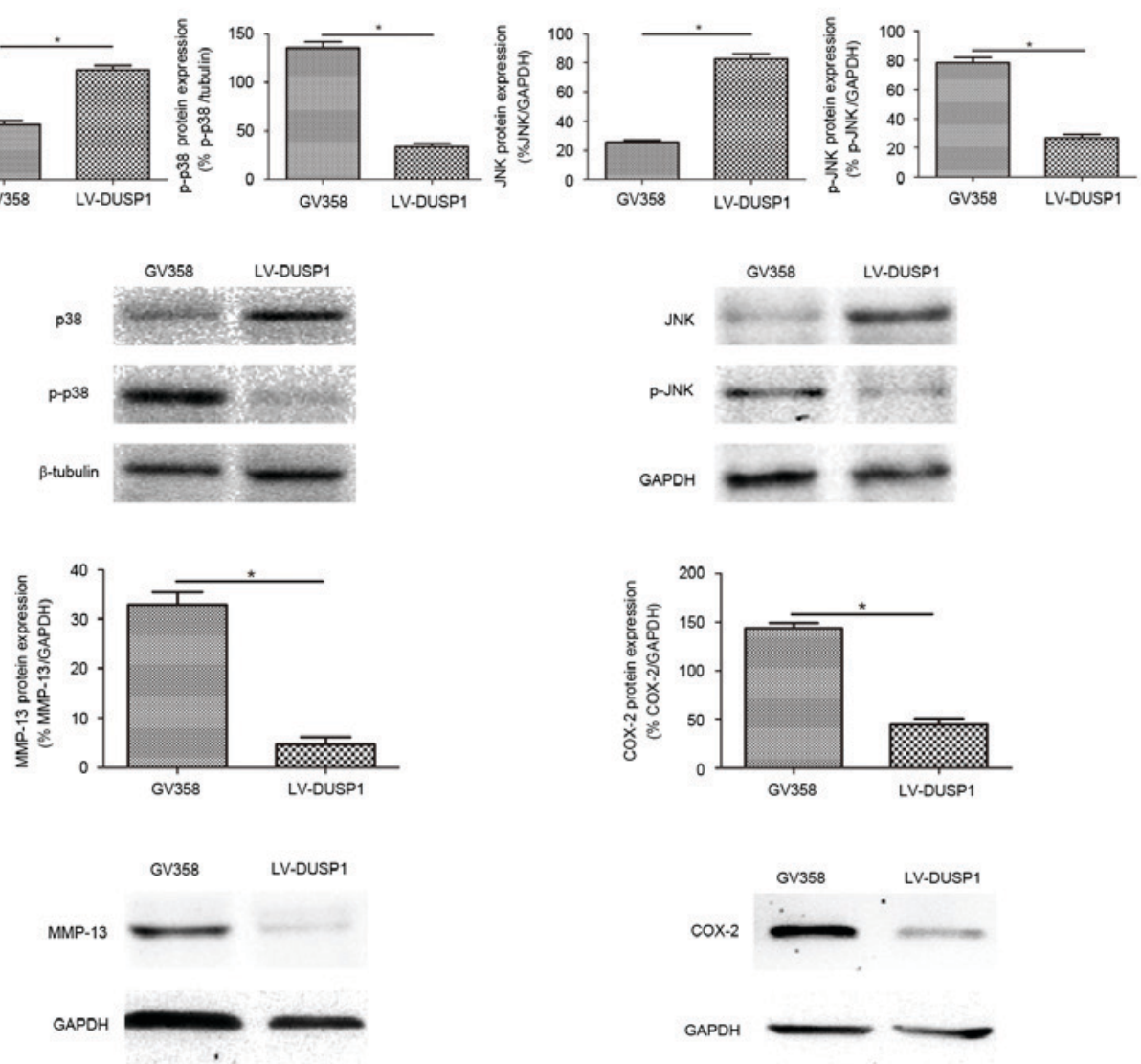

Figure 3. Effect of DUSP1 overexpression in osteoarthritis FLS. (A) Fluorescence microscopy and light microscopy images of FLS. The infection efficiency of GV358 (control lentivirus vector) and LV-DUSP1 (GV358 combined with DUSP1 gene) was almost 90\% (magnification, x200). (B) Expression of DUSP1 was increased by LV-DUSP1. (C) Activation of p38 mitogen-activated protein kinase and JNK pathway was inhibited in the presence of LV-DUSP1. (D) Overexpression of DUSP1 decreased the expression of COX-2 and MMP-13. Student's t test was performed for the comparison of the results. Data are presented as the mean \pm standard error $(n=3)$. * $P<0.01$. FLSs, fibroblast-like synoviocytes; GV358, control vector; LV, lentivirus; DUSP1, dual specificity phosphatase1; p-, phosphorylated; JNK, c-Jun NH2 terminal kinase; MMP-13, matrix metalloproteinase-13; COX-2, cyclooxygenase-2.

various inflammatory mediators and have been successfully used in the treatment of inflammatory diseases for many years (34). Dex was used to induce OA FLSs in this current study, and the results suggested that Dex induced the expression of DUSP1 at the mRNA and protein level in OA FLSs. This result is consistent with the literature data indicating that Dex is an important regulator of DUSP1 $(20,34,35)$.

It has been known that MAPK signaling pathway has a critical role in the regulation of inflammatory and catabolic mediators, such as COX-2 and MMP-13 (36,37). In OA,
MMP-13 and COX-2 have a critical role in maintaining cartilage homeostasis $(15,38)$. MMP-13 is involved in type II collagen cleavage, which contributes to the degradation of joint cartilage. COX-2 is likely responsible for the elevated prostaglandin $\mathrm{E} 2$, which has a key role in OA progression and pain (39). Furthermore, the activation of p38 MAPK and JNK pathway, and the expression of MMP-13 and COX-2 were investigated in the three groups of FLSs in the present study. As illustrated in the results, p38 MAPK and JNK were significantly activated and the expression of MMP-13 and COX-2 in OA FLSs were higher than the other two groups cells. These 
findings suggest that Dex can inhibit the activation of MAPKs, and exhibit anti-inflammatory and anti-catabolic effects in OA FLSs, and the effects of Dex may be dependent on the induction of DUSP1; the specific evidence requires further investigation.

Additionally, to elucidate the role of DUSP1 in OA FLSs, LV-DUSP1 and GV358 were constructed to infect OA FLSs, and the activation of the MAPK pathway, and the expression of MMP-13 and COX-2 were detected. The results demonstrated that the activated MAPKs were inhibited in the presence of LV-DUSP1 in OA FLSs, and the expression levels of OA-associated mediators, MMP-13 and COX-2, were decreased. The findings are consistent with the role of DUSP1 as a negative regulator of the MAPK pathway, and suggest that DUSP1 has an anti-inflammatory role in certain inflammation-associated diseases.

In conclusion, the results demonstrated that the expression of DUSP1 was lower in OA FLSs than in normal FLSs, and DUSP1 may inhibit the expression of OA-associated mediators, MMP-13 and COX-2, by suppressing the activation of p38 MAPK and JNK pathways in OA FLSs. In addition, the anti-inflammatory role of Dex may partially depend on induction of DUSP1. The findings of the present study suggest that DUSP1 may be a promising therapeutic target in $\mathrm{OA}$.

\section{Acknowledgements}

This work was supported by Institute of Osteosarcoma, Tangdu Hospital, The Fourth Military Medical University (Xi'an, China).

\section{References}

1. Cross M, Smith E, Hoy D, Nolte S, Ackerman I, Fransen M, Bridgett L, Williams S, Guillemin F, Hill CL, et al: The global burden of hip and knee osteoarthritis: Estimates from the global burden of disease 2010 study. Ann Rheum Dis 73: 1323-1330, 2014.

2. Dieppe PA and Lohmander LS: Pathogenesis and management of pain in osteoarthritis. Lancet 365: 965-973, 2005.

3. Goldring MB and Otero M: Inflammation in osteoarthritis. Curr Opin Rheumatol 23: 471-478, 2011.

4. Berenbaum F: Osteoarthritis as an inflammatory disease (osteoarthritis is not osteoarthrosis!). Osteoarthritis Cartilage 21: 16-21, 2013.

5. Ayral X, Pickering EH, Woodworth TG, Mackillop N and Dougados M: Synovitis: A potential predictive factor of structural progression of medial tibiofemoral knee osteoarthritis - results of a 1 year longitudinal arthroscopic study in 422 patients Osteoarthritis Cartilage 13: 361-367, 2005.

6. Benito MJ, Veale DJ, FitzGerald O, van den Berg WB and Bresnihan B: Synovial tissue inflammation in early and late osteoarthritis. Ann Rheum Dis 64: 1263-1267, 2005.

7. Scanzello CR, McKeon B, Swaim BH, DiCarlo E, Asomugha EU, Kanda V, Nair A, Lee DM, Richmond JC, Katz JN, et al: Synovial inflammation in patients undergoing arthroscopic meniscectomy: Molecular characterization and relationship to symptoms. Arthritis Rheum 63: 391-400, 2011.

8. Chang L and Karin C: Mammalian MAP kinase signalling cascades. Nature 410: 37-40, 2001.

9. Johnson GL and Lapadat R: Mitogen-activated protein kinase pathways mediated by ERK, JNK, and p38 protein kinases. Science 298: 1911-1912, 2002.

10. Rincón M and Davis RJ: Regulation of the immune response by stress-activated protein kinases. Immunol Rev 228: 212-224, 2009.
11. Cuenda A and Rousseau S: p38 MAP-kinases pathway regulation, function and role in human diseases. Biochim Biophys Acta 1773: 1358-3675, 2007.

12. Fechir M, Linker K, Pautz A, Hubrich T, Förstermann U, Rodriguez-Pascual $\mathrm{F}$ and Kleinert $\mathrm{H}$ : Tristetraprolin regulates the expression of the human inducible nitric-oxide synthase gene. Mol Pharmacol 67: 2148-2161, 2005.

13. Ashwell JD: The many paths to p38 mitogen-activated protein kinase activation in the immune system. Nat Rev Immunol 6: 532-540, 2006.

14. Ono $\mathrm{K}$ and Han J: The p38 signal transduction pathway: Activation and function. Cell Signal 12: 1-13, 2000.

15. Zeng L, Wang W, Rong XF, Zhong Y, Jia P, Zhou GQ and Li RH: Chondroprotective effects and multi-target mechanisms of Icariin in IL-1 beta-induced human SW 1353 chondrosarcoma cells and a rat osteoarthritis model. Int Immunopharmacol 18: 175-181, 2014.

16. Zhao Q, Shepherd EG, Manson ME, Nelin LD, Sorokin A and Liu Y: The role of mitogen-activated protein kinase phosphatase-1 in the response of alveolar macrophages to lipopolysaccharide: Attenuation of proinflammatory cytokine biosynthesis via feedback control of p38. J Biol Chem 280: 8101-8108, 2005.

17. Chi H, Barry SP, Roth RJ, Wu JJ, Jones EA, Bennett AM and Flavell RA: Dynamic regulation of pro- and anti-inflammatory cytokines by MAPK phosphatase 1 (MKP-1) in innate immune responses. Proc Natl Acad Sci USA 103: 2274-2279, 2006.

18. Liu Y, Shepherd EG and Nelin LD: MAPK phosphatases-regulating the immune response. Nat Rev Immunol 7: 202-212, 2007.

19. Boutros T, Chevet E and Metrakos P: Mitogen-activated protein (MAP) kinase/MAP kinase phosphatase regulation: Roles in cell growth, death, and cancer. Pharmacol Rev 60: 261-310, 2008

20. Kassel O, Sancono A, Krätzschmar J, Kreft B, Stassen M and Cato AC: Glucocorticoids inhibit MAP kinase via increased expression and decreased degradation of MKP-1. EMBO J 20: 7108-7116, 2001.

21. Nieminen R, Korhonen R, Moilanen T, Clark AR and Moilanen E: Aurothiomalate inhibits cyclooxygenase 2, matrix metalloproteinase 3 , and interleukin- 6 expression in chondrocytes by increasing MAPK phosphatase 1 expression and decreasing p38 phosphorylation: MAPK phosphatase 1 as a novel target for antirheumatic drugs. Arthritis Rheum 62: 1650-1659, 2010.

22. McAbee J, Li Q, Yu H and Kirkwood KL: Sexual dimorphism in periapical inflammation and bone loss from mitogen-activated protein kinase phosphatase-1 deficient mice. J Endod 38: 1097-1100, 2012.

23. Hashizume M and Mihara M: High molecular weight hyaluronic acid inhibits IL-6-induced MMP production from human chondrocytes by up-regulating the ERK inhibitor, MKP-1. Biochem Biophys Res Commun 403: 184-189, 2010.

24. Smallie T, Ross EA, Ammit AJ, Cunliffe HE, Tang T, Rosner DR, Ridley ML, Buckley CD, Saklatvala J, Dean JL and Clark AR: Dual-specificity phosphatase 1 and tristetraprolin cooperate to regulate macrophage responses to lipopolysaccharide. J Immunol 195: 277-288, 2015.

25. Matta R, Barnard JA, Wancket LM, Yan J, Xue J, Grieves J, Frazier WJ, Nelin L, Cato AC and Liu Y: Knockout of Mkp-1 exacerbates colitis in Il-10-deficient mice. Am J Physiol Gastrointest Liver Physiol 302: G1322-G1335, 2012.

26. Vattakuzhi Y, Abraham SM, Freidin A, Clark AR and Horwood NJ: Dual-specificity phosphatase 1-null mice exhibit spontaneous osteolytic disease and enhanced inflammatory osteolysis in experimental arthritis. Arthritis Rheum 64: 2201-2210, 2012.

27. Lattuada D, Gualtierotti R, Crotta K, Seneci P, Ingegnoli F, Corradini C, Viganò R, Marelli $\mathrm{O}$ and Casnici C: Smac127 has proapoptotic and anti-inflammatory effects on rheumatoid arthritis fibroblast-like synoviocytes. Mediators Inflamm 2016: 6905678,2016

28. Livak KJ and Schmittgen TD: Analysis of relative gene expression data using real-time quantitative PCR and the 2(-Delta Delta C(T)) method. Methods 25: 402-408, 2001.

29. Buckwalter JA, Mankin HJ and Grodzinsky AJ: Articular cartilage and osteoarthritis. Instr Course Lect 54: 465-480, 2005.

30. Laev SS and Salakhutdinov NF: Anti-arthritic agents: Progress and potential. Bioorg Med Chem 23: 3059-3080, 2015.

31. Lawan A, Shi H, Gatzke F and Bennett AM: Diversity and specificity of the mitogen-activated protein kinase phosphatase-1 functions. Cell Mol Life Sci 70: 223-237, 2013. 
32. Wancket LM, Frazier WJ and Liu Y: Mitogen-activated protein kinase phosphatase (MKP)-1 in immunology, physiology, and disease. Life Sci 90: 237-248, 2012.

33. Mahalingam CD, Datta T, Patil RV, Kreider J, Bonfil RD, Kirkwood KL, Goldstein SA, Abou-Samra AB and Datta NS: Mitogen-activated protein kinase phosphatase 1 regulates bone mass, osteoblast gene expression, and responsiveness to parathyroid hormone. J Endocrinol 211: 145-156, 2011.

34. Newton R: Molecular mechanisms of glucocorticoid action: What is important? Thorax 55: 603-613, 2000.

35. Abraham SM, Lawrence T, Kleiman A, Warden P, Medghalchi M, Tuckermann J, Saklatvala J and Clark AR: Antiinflammatory effects of dexamethasone are partly dependent on induction of dual specificity phosphatase 1. J Exp Med 203: 1883-1889, 2006.
36. Mariani E, Pulsatelli L and Facchini A: Signaling pathways in cartilage repair. Int J Mol Sci 15: 8667-8698, 2014.

37. Amin AR, Attur M, Patel RN, Thakker GD, Marshall PJ, Rediske J, Stuchin SA, Patel IR and Abramson SB: Superinduction of cyclooxygenase-2 activity in human osteoarthritis-affected cartilage. Influence of nitric oxide. J Clin Invest 99: 1231-1237, 1997.

38. Ying X, Peng L, Chen H, Shen Y, Yu K and Cheng S: Cordycepin prevented IL- $\beta$-induced expression of inflammatory mediators in human osteoarthritis chondrocytes. Int Orthop 38: 1519-1526, 2014.

39. Lee AS, Ellman MB, Yan D, Kroin JS, Cole BJ, van Wijnen AJ and Im HJ: A current review of molecular mechanisms regarding osteoarthritis and pain. Gene 527: 440-447, 2013. 